\title{
THE HOPF TYPE THEOREM FOR EQUIVARIANT LOCAL MAPS
}

\author{
PIOTR BARTŁOMIEJCZYK
}

\begin{abstract}
We study otopy classes of equivariant local maps and prove the Hopf type theorem for such maps in the case of a real finite dimensional orthogonal representation of a compact Lie group.
\end{abstract}

\section{INTRODUCTION}

The famous Hopf theorem states that if $M$ is an $n$-dimensional connected orientable closed manifold, $S^{n}$ the $n$-dimensional sphere and $\left[M, S^{n}\right]$ denotes the set of homotopy classes of continuous maps from $M$ to $S^{n}$, then the Brouwer degree map deg: $\left[M, S^{n}\right] \rightarrow \mathbb{Z}$ is a bijection for $n \geq 1$.

Our purpose is to prove a natural equivariant version of this theorem. More precisely, in our version we replace the set of homotopy classes by the set of otopy classes and we take into account the group action. Namely, let $V$ be a real finite dimensional orthogonal representation of a compact Lie group $G, \Omega$ be an open invariant subset of $V$ and $\mathcal{F}_{G}[\Omega]$ denote the set of equivariant otopy classes of equivariant local maps with open invariant domains contained in $\Omega$. Assume that $0 \notin \Omega$ or $\operatorname{dim} V^{G}>0$. Our main result states that there is a natural bijection

$$
\mathcal{F}_{G}[\Omega] \approx \prod_{(H)}\left(\sum_{i=1}^{n(H)} \mathbb{Z}\right),
$$

where the product (finite) extends over the orbit types $(H)$ in $\Omega$ satisfying $\operatorname{dim} W H=0$, where $W H$ is the Weyl group of $H$, and the direct sums (finite or countably infinite) are taken over the sets of all connected components of the quotients $\Omega_{H} / W H$.

The proof of our main theorem follows from the following two results: the splitting result which gives a product decomposition of $\mathcal{F}_{G}[\Omega]$ with respect to the orbit types $(H)$ in $\Omega$ satisfying $\operatorname{dim} W H=0$ (proved in [1] and [4]) and the full description of each factor of the above product decomposition (proved here). In turn, the proof of the second above result is based on the

Date: September 20, 2021.

2010 Mathematics Subject Classification. Primary: 55P91; Secondary: 54C35.

Key words and phrases. Hopf theorem, equivariant map, otopy, topological degree. 
classification of fiber otopy classes of local cross sections of a vector bundle and establishing (in case of free actions) a bijection between the set of otopy classes of equivariant local maps and the set of fiber otopy classes of local cross sections of some vector bundle.

Our paper contains also some remarks concerning the parametrized case. Although the consideration of the parameters may seem to be an artificial generalization, but, in fact, it is a natural way to study the higher homotopy groups of the space $\mathcal{F}_{G}(\Omega)$ using the formula $\mathcal{F}_{G}\left[\mathbb{R}^{k} \times \Omega\right] \approx \pi_{k}\left(\mathcal{F}_{G}(\Omega)\right)$ established in [1].

The organization of the paper is as follows. Section 1 presents some preliminaries. In Section 2 we introduce the space of local cross sections of a vector bundle and state two results concerning the classification of fiber otopy classes of such sections. Section 3 contains the proof of the second of these results. In Section 4 we give the description of the set of otopy classes of equivariant local maps in case of free actions. Finally, in Section 5 we prove our main result i.e. the Hopf type theorem for equivariant local maps.

\section{Preliminaries}

The notation $A \Subset B$ means that $A$ is a compact subset of $B$. For a topological space $X$, let $\tau(X)$ denote the topology on $X$. Recall that if $A$, $B$ are topological spaces, then $\operatorname{Map}(A, B)$ denotes the set of all continuous maps of $A$ into $B$ equipped with the usual compact-open topology i.e. having as subbasis all the sets $\Gamma(C, U)=\{f \in \operatorname{Map}(A, B) \mid f(C) \subset U\}$ for $C \Subset A$ and $U$ open in $B$.

For any topological spaces $X$ and $Y$, let $\mathcal{M}(X, Y)$ be the set of all continuous maps $f: D_{f} \rightarrow Y$ such that $D_{f}$ is an open subset of $X$. Let $\mathcal{R}$ be a family of subsets of $Y$. We define

$$
\operatorname{Loc}(X, Y, \mathcal{R}):=\left\{f \in \mathcal{M}(X, Y) \mid f^{-1}(R) \Subset D_{f} \text { for all } R \in \mathcal{R}\right\} .
$$

We introduce a topology in $\operatorname{Loc}(X, Y, \mathcal{R})$ generated by the subbasis consisting of all sets of the form

- $H(C, U):=\left\{f \in \operatorname{Loc}(X, Y, \mathcal{R}) \mid C \subset D_{f}, f(C) \subset U\right\}$ for $C \Subset X$ and $U \in \tau(Y)$,

- $M(V, R):=\left\{f \in \operatorname{Loc}(X, Y, \mathcal{R}) \mid f^{-1}(R) \subset V\right\}$ for $V \in \tau(X)$ and $R \in \mathcal{R}$.

Elements of $\operatorname{Loc}(X, Y, \mathcal{R})$ are called local maps. The natural base point of $\operatorname{Loc}(X, Y, \mathcal{R})$ is the empty map. The set-theoretic union of two local maps $f$ and $g$ with disjoint domains will be denoted by $f \sqcup g$. We define the space of proper maps $\operatorname{Prop}(X, Y)$ to be $\operatorname{Loc}(X, Y, \mathcal{K})$, where $\mathcal{K}=\{K \mid K \Subset Y\}$. 
Moreover, in the case when $\mathcal{R}=\{\{y\}\}$ we will write $\operatorname{Loc}(X, Y, y)$ omitting double curly brackets.

Assume that $G$ is a topological group and $X, Y$ are two $G$-spaces. We will denote by $\operatorname{Map}_{G}(X, Y)$ the subset of $\operatorname{Map}(X, Y)$ consisting of all maps $f$ such that

$$
f(g x)=g f(x) \text { for all } x \in X \text { and } g \in G
$$

endowed with the relative topology. Elements of $\operatorname{Map}_{G}(X, Y)$ are called equivariant maps or $G$-maps. Let $\operatorname{Loc}_{G}(X, Y, \mathcal{R})\left(\operatorname{resp} \operatorname{Prop}_{G}(X, Y)\right)$ be the subspace of $\operatorname{Loc}(X, Y, \mathcal{R})$ (resp. $\operatorname{Prop}(X, Y))$ consisting of equivariant maps with invariant domains and equipped with the induced topology. Elements of $\operatorname{Loc}_{G}(X, Y, \mathcal{R})$ will be called equivariant local maps or local $G$-maps.

Assume that $V$ is a real finite dimensional orthogonal representation of a compact Lie group $G$. Throughout the paper $\mathbb{R}^{k}$ denotes a trivial representation of $G$. Let $\Omega$ be an open invariant subset of $\mathbb{R}^{k} \oplus V$. Let us introduce the following notation:

$$
\begin{aligned}
& \mathcal{F}_{G}(\Omega):=\operatorname{Loc}_{G}(\Omega, V, 0), \\
& \mathcal{P}_{G}(\Omega):=\operatorname{Prop}_{G}(\Omega, V) .
\end{aligned}
$$

Let $I=[0,1]$. We assume that the action of $G$ on $I$ is trivial. Any element of $\operatorname{Loc}_{G}(I \times \Omega, V, 0)$ is called an otopy and any element of $\operatorname{Prop}_{G}(I \times \Omega, V)$ is called a proper otopy. In [1] we proved that each otopy (resp. proper otopy) corresponds to a path in $\mathcal{F}_{G}(\Omega)\left(\operatorname{resp} . \mathcal{P}_{G}(\Omega)\right)$ and vice versa.

Given a (proper) otopy $h: \Lambda \subset I \times \Omega \rightarrow V$ we can define for each $t \in I$ sets $\Lambda_{t}=\{x \in \Omega \mid(t, x) \in \Lambda\}$ and maps $h_{t}: \Lambda_{t} \rightarrow V$ with $h_{t}(x)=h(t, x)$. Note that from the above $h_{t}$ may be the empty map. If $h$ is a (proper) otopy, we say that $h_{0}$ and $h_{1}$ are (proper) otopic. Of course, (proper) otopy gives an equivalence relation on $\mathcal{F}_{G}(\Omega)\left(\mathcal{P}_{G}(\Omega)\right)$. The set of (proper) otopy classes will be denoted by $\mathcal{F}_{G}[\Omega]\left(\mathcal{P}_{G}[\Omega]\right)$. In [1, Prop. 2.4] we showed that the map $\mathcal{P}_{G}[\Omega] \rightarrow \mathcal{F}_{G}[\Omega]$ induced by the inclusion is a bijection. Observe that if $f \in \mathcal{F}_{G}(\Omega)$ and $V$ is an open invariant subset of $D_{f}$ such that $f^{-1}(0) \subset V$, then $f$ and $\left.f\right|_{V}$ are otopic. In particular, if $f^{-1}(0)=\emptyset$ then $f$ is otopic to the empty map.

Recall that every Lie group is orientable. Let $G$ be a compact Lie group. For any $g \in G$ we will denote by $A_{g}: G \rightarrow G$ the automorphism given by $A_{g}(h)=g h g^{-1}$ for all $h \in G$. Let $T_{e} G$ denote the tangent space at the unit element. We say that $G$ is biorientable if for each $g \in G$ the derivative $D A_{g}(e): T_{e} G \rightarrow T_{e} G$ preserves the orientation. It is not hard to show that if $G$ is abelian, finite or has an odd number of connected components, then $G$ is 
biorientable. The group $O(2)$ seems to be the simplest example of a compact Lie group which is not biorientable. Let $V$ be a real finite dimensional representation of a group $G$. We say that the action of $G$ is orientationpreserving if for each $g \in G$ the map $v \mapsto g \cdot v$ preserves the orientation of $V$. In Section 4 we will need the following result, which can be found in $[5$, Lem. 3.4].

Lemma 1.1. Assume $V$ is a real finite dimensional orthogonal representation of a compact Lie group $G$ and $U$ is an open invariant subset of $V$ on which $G$ acts freely. If $G$ is biorientable and the action of $G$ is orientationpreserving then $U / G$ is orientable.

Remark 1.2. If $G$ is not biorientable then $U / G$ may be orientable or not, or even may consist of many components, of which some are orientable and some are not.

Assume $V$ is a real finite dimensional orthogonal representation of a compact Lie group $G$ and $H$ is a closed subgroup of $G$. Recall that $G_{x}=$ $\{g \in G \mid g x=x\},(H)$ stands for a conjugacy class of $H$ and $W H=N H / H$, where $N H$ is a normalizer of $H$ in $G$. Let $\Omega$ be an open invariant subset of $V$. We define the following subsets of $\Omega$ :

$$
\begin{aligned}
\Omega^{H} & =\left\{x \in \Omega \mid H \subset G_{x}\right\}, \\
\Omega_{H} & =\left\{x \in \Omega \mid H=G_{x}\right\}, \\
\Omega_{(H)} & =\left\{x \in X \mid(H)=\left(G_{x}\right)\right\} .
\end{aligned}
$$

Let

$$
\begin{aligned}
\Phi(G) & =\{(H) \mid H \text { is a closed subgroup of } G\}, \\
\operatorname{Iso}(\Omega) & =\left\{(H) \in \Phi(G) \mid \Omega_{(H)} \neq \emptyset\right\} .
\end{aligned}
$$

The set $\operatorname{Iso}(\Omega)$ is partially ordered. Namely, $(H) \leq(K)$ if $H$ is conjugate to a subgroup of $K$.

Throughout the paper we will make use of the following well-known facts:

- $W H$ is a compact Lie group,

- $V^{H}$ is a linear subspace of $V$ and orthogonal representation of $W H$,

- the action of $W H$ on $\Omega_{H}$ is free,

- $\Omega_{H}$ is open and dense in $\Omega^{H}$.

\section{The SPACE OF LOCAL CROSS SECTIONS OF A VECTOR BUNDLE}

All manifolds considered are without boundary. Assume $p: E \rightarrow M$ is a smooth (i.e., $C^{1}$ ) vector bundle. We will identify $M$ with the zero section 
of $E$. A local cross section of a bundle $p: E \rightarrow M$ is a continuous map $s: U \rightarrow E$, where $U$ is open in $M, s^{-1}(M)$ is compact and $p \circ s=\operatorname{id}_{U}$. Let $\Gamma_{\text {loc }}(M, E)$ denote the set of all local cross sections of $E$ over $M$. A fiber otopy is a continuous map $h: \Lambda \rightarrow E$ such that $\Lambda$ is open in $I \times M, h^{-1}(M)$ is compact and $p(h(t, x))=x$ for all $(t, x) \in \Lambda$. Let $s, s^{\prime} \in \Gamma_{\text {loc }}(M, E)$. We say that $s$ and $s^{\prime}$ are fiber otopic provided there is a fiber otopy $h$ such that $h_{0}=s$ and $h_{1}=s^{\prime}$, where $h_{t}(x)=h(t, x)$. Of course, fiber otopy gives an equivalence relation on $\Gamma_{\text {loc }}(M, E)$, which will be denoted by $s \sim s^{\prime}$. Let $\Gamma_{\text {loc }}[M, E]$ denote the set of fiber otopy classes of local cross sections of $p: E \rightarrow M$. Observe that if $s$ is a local cross section and $V$ is an open subset of $D_{s}$ such that $s^{-1}(M) \subset V$, then $s$ and $\left.s\right|_{V}$ are fiber otopic. This property of local cross section will be called localization. In particular, if $s^{-1}(M)=\emptyset$ then $s$ is fiber otopic to the empty map. The set-theoretic union of two local cross sections $s$ and $s^{\prime}$ with disjoint domains will be denoted by $s \sqcup s^{\prime}$.

The following result has been proved in [1, Prop. 3.2].

Proposition 2.1. If $\operatorname{rank} E>\operatorname{dim} M$ then $\Gamma_{l o c}[M, E]$ has a single element.

In the remainder of this and in the next section we assume that rank $E=$ $\operatorname{dim} M$. Let us denote by $\mathrm{I}_{2}(s)$ the mod 2 intersection number and by $\mathrm{I}(s)$ the oriented intersection number of a local cross section $s$ with the zero section (see for instance [6]). It is evident that in both cases the intersection number is otopy invariant i.e. if two local cross sections are fiber otopic then they have the same intersection number, but more interestingly, the converse is also true. Namely, the following result, which may be viewed as a version of the well-known Hopf theorem, will be proved in the next section.

Theorem 2.2. Let $M$ be connected.

(1) If $E$ is orientable as a manifold then $\mathrm{I}: \Gamma_{\text {loc }}[M, E] \rightarrow \mathbb{Z}$ is a bijection.

(2) If $E$ is non-orientable as a manifold then $\mathrm{I}_{2}: \Gamma_{\text {loc }}[M, E] \rightarrow \mathbb{Z}_{2}$ is a bijection.

Remark 2.3. Observe that if $E=T M$ then local cross sections are local vector fields. Let $\mathcal{V}_{\text {loc }}[M]:=\Gamma_{\text {loc }}[M, T M]$. Since $T M$ is always orientable as a manifold we obtain that $\mathrm{I}: \mathcal{V}_{\text {loc }}[M] \rightarrow \mathbb{Z}$ is a bijection for $M$ smooth connected.

Remark 2.4. For now, we do not have any satisfactory description of $\Gamma_{\text {loc }}[M, E]$ if $\operatorname{rank} E<\operatorname{dim} M$. 


\section{Proof of Theorem 2.2}

We have divided the proof of Theorem 2.2 into a sequence of lemmas and propositions. We will need the following transversality result for sections of a smooth vector bundle, which can be easily derived from the transversality theorem for maps. A smooth local cross section is called generic if it is transverse to the zero section.

Lemma 3.1. Arbitrarily close (in the $C^{1}$ sense) to any smooth local cross section of a smooth vector bundle there exists a generic local cross section (both have the same domain).

Let us denote by $B(p ; r)$ the open $r$-ball in $M$ around $p$. A generic local cross section $s$ is called standard if $D_{s}=B(p ; r)$ and $s^{-1}(M)=\{p\}$. The proof of Theorem 2.2 is based on the following propositions and lemmas.

Proposition 3.2. Any local cross section is otopic to a finite disjoint union of standard ones.

Proof. By Lemma 3.1, any local cross section is otopic (also homotopic) to generic one and by localization property, any generic local cross section is otopic to a finite disjoint union of standard ones.

Let us state three lemmas concerning standard local cross sections and needed below. We omit their proofs since they either follow directly from definitions or are very similar to those from the topological degree theory (see for example [3]). In the following lemmas we assume that all considered local cross sections are defined on the ball $B(p ; r)$. Moreover, assume that there are a local trivialization $\psi: U \times \mathbb{R}^{n} \rightarrow E$ and a coordinate system $\phi: W \subset M \rightarrow \mathbb{R}^{n}$ such that $B(p ; r) \subset U \cap W$ and $\phi(p)=0$. Of course, if $r$ is small enough such a trivialization and a coordinate system always exist. Furthermore, under the above assumptions any standard local cross section has the form $s(x)=\psi(x, f(\phi(x)))$ with $f: \phi(B(p ; r)) \subset \mathbb{R}^{n} \rightarrow \mathbb{R}^{n}$ smooth. Finally, observe that $s_{M}(x)=\psi(x, M \phi(x))$ is a standard local cross section for any $M \in \mathrm{Gl}_{n}(\mathbb{R})$.

Lemma 3.3. Let $A \in \mathrm{Gl}_{n}(\mathbb{R})$.

(1) If $E$ is orientable as a manifold then $\mathrm{I}\left(s_{A}\right)=\operatorname{sgn} \operatorname{det} A$.

(2) If $E$ is non-orientable as a manifold then $\mathrm{I}_{2}\left(s_{A}\right)=1$.

Lemma 3.4. Let $A, B \in \mathrm{Gl}_{n}(\mathbb{R})$.

(1) If $E$ is orientable as a manifold and $\operatorname{sgn} \operatorname{det} A=\operatorname{sgn} \operatorname{det} B$ then $s_{A}$ and $s_{B}$ are fiber otopic. 
(2) If $E$ is non-orientable as a manifold then $s_{A}$ and $s_{B}$ are fiber otopic.

Lemma 3.5. If $s(x)=\psi(x, f(\phi(x)))$ is a standard local cross section then so is $\widetilde{s}(x)=\psi(x, D f(0) \phi(x))$ and there is $R \in(0, r)$ such that the straightline homotopy between $s$ and $\widetilde{s}$ on $B(p ; R)$ is a fiber otopy.

The above map $\widetilde{s}$ will be called a linearization of the standard local cross section $s$. The next result follows easily from Lemmas 3.3 and 3.5.

Corollary 3.6. Let $s$ be a standard local cross section.

(1) If $E$ is orientable as a manifold then $|\mathrm{I}(s)|=1$.

(2) If $E$ is non-orientable as a manifold then $\mathrm{I}_{2}(s)=1$.

Proposition 3.7. Let $s$ and $s^{\prime}$ be standard local cross sections.

(1) If $E$ is orientable as a manifold and $\mathrm{I}(s)=\mathrm{I}\left(s^{\prime}\right)$ then $s \sim s^{\prime}$.

(2) If $E$ is non-orientable as a manifold then $s \sim s^{\prime}$.

Proof. Step 1. Observe that any standard local cross section can be "translated" from one point on the manifold $M$ to another using a finite sequence of translations in images of coordinate systems. Such a "translation" is not unique, but it is always fiber otopic to the original local cross section. So without loss of generality we can assume that $s$ and $s^{\prime}$ are defined on the same ball.

Step 2. By Lemma 3.5 two standard local cross sections defined on the same ball are fiber otopic to their linearizations and by Lemma 3.4 these linearizations are fiber otopic, which completes the proof.

Remark 3.8. In fact, in the orientable case, we will need the following slight generalization of Proposition 3.7. Consider two finite sequences $\left\{s_{i}\right\}_{1}^{n}$ and $\left\{s_{i}^{\prime}\right\}_{1}^{n}$ of standard local cross sections such that for each sequence the domains of local cross sections are pairwise disjoint and all $2 n$ sections have the same intersection number. Then $\sqcup_{i=1}^{n} s_{i} \sim \sqcup_{i=1}^{n} s_{i}^{\prime}$. To verify our claim, observe that, the procedure described in Step 1 of the proof of Proposition 3.7 can be done simultaneously for all $s_{i}$ in such a way that their domains remain pairwise disjoint during the "translation".

Proposition 3.9 (Annihilation). Assume that $s$ and $s^{\prime}$ are standard local cross sections with disjoint domains.

(1) If $E$ is orientable as a manifold and $\mathrm{I}(s)=-\mathrm{I}\left(s^{\prime}\right)$ then $s \sqcup s^{\prime} \sim \emptyset$.

(2) If $E$ is non-orientable as a manifold then $s \sqcup s^{\prime} \sim \emptyset$.

Proof. As in Step 1 from the previous proof we can "move" one of standard local cross sections and, in consequence, we can assume that the domains of 
$s$ and $s^{\prime}$ are contained in the same trivialization $\psi$ and coordinate system $\phi$. Let $D_{s}=B(p ; r)$ and $D_{s^{\prime}}=B(q ; r)$. By Lemma $3.5 s$ and $s^{\prime}$ are fiber otopic to their linearizations and by Lemma 3.4 these linearizations are fiber otopic to $s_{A}(x)=\psi(x, A(\phi(x)-\phi(p)))$ and $s_{B}(x)=\psi(x, B(\phi(x)-\phi(q)))$, where $A$ is an identity and $B$ is a symmetry. Finally, repeating the procedure from the proof of Lemma 3.2 in [2] we obtain that $s_{A}$ and $s_{B}$ annihilate that is $s_{A} \sqcup s_{B} \sim \emptyset$.

The following result is an easy consequence of Propositions 3.2 and 3.9.

Proposition 3.10. Let $s$ be a local cross section.

(1) If $E$ is orientable as a manifold and $\mathrm{I}(s)=m$ then $s$ is otopic to a disjoint union of $|m|$ standard local cross sections, each of them with the same intersection number equal to 1 (resp. -1) if $m \geq 0$ (resp. $m<0)$.

(2) If $E$ is non-orientable as a manifold and $\mathrm{I}_{2}(s)=m$ then $s$ is otopic to $m$ standard local cross sections.

Proof of Theorem 2.2. Surjectivity follows easily from Lemma 3.3. In turn, injectivity in the orientable case follows immediately from Proposition 3.10(1) and Remark 3.8 and in the non-orientable case from Proposition 3.10(2) and Proposition 3.7(2).

\section{The Set of otopy Classes in CASE of FREE ACTIONS}

Assume that $V$ is a real finite dimensional orthogonal representation of a compact Lie group $G$. Let $\Omega$ be an open invariant subset of $V, G$ act freely on $\Omega, M:=\Omega / G, E:=(\Omega \times V) / G$. The trivial vector bundle $\Omega \times V \rightarrow \Omega$ factorizes to the vector bundle $p: E \rightarrow M$ with the typical fiber $F=\mathbb{R}^{n}$. In [1] we proved the following result.

Theorem 4.1. The function $\Phi: \mathcal{F}_{G}(\Omega) \rightarrow \Gamma_{l o c}(M, E)$ given by $\Phi(f):=$ $s_{f}$, where $s_{f}([x]):=[x, f(x)]$, is bijective. Moreover, $\Phi$ induces a bijection between $\mathcal{F}_{G}[\Omega]$ and $\Gamma_{\text {loc }}[M, E]$.

Remark 4.2. Since $\Phi$ is a bijection, $\Gamma_{\text {loc }}(M, E)$ is given the induced topology, in which $\Phi$ is a homeomorphism. Moreover, the topology on $\Gamma_{\text {loc }}(M, E)$ coincides with the relative topology induced from $\operatorname{Loc}(M, E,\{M\})$. Finally, both otopies and fiber otopies correspond to paths in the respective spaces and vice versa.

Recall that if $\operatorname{dim} G>0$ then the set $\mathcal{F}_{G}[\Omega]$ has a single element (see [1, Theorem 3.1]). The next result is devoted to the case $\operatorname{dim} G=0$. Note 
that the set of connected components of the quotient $\Omega / G$ is either finite or countably infinite.

Theorem 4.3. If $G$ is zero-dimensional (i.e. finite) then there is a natural bijection

$$
\mathcal{F}_{G}[\Omega] \approx \sum_{\alpha} \mathbb{Z}
$$

where the direct sum is taken over the set of all connected components $\alpha$ of the quotient $\Omega / G$.

Proof. If $\operatorname{dim} G=0$ then $\operatorname{rank} E=\operatorname{dim} M$. Moreover, by Theorem 1.1, $E$ is orientable as a manifold. Now the assertion is an easy consequence of Theorems 2.2 and 4.1. Observe that a direct sum (not product) appears in the right-hand side of (4.1), since for any local cross section $s$ the preimage $s^{-1}(M)$ meets only a finite number of components of $M$.

We close this section with some remarks concerning the parametrized case. Assume that $\Omega$ is an open invariant subset of $\mathbb{R}^{k} \oplus V$ with trivial action of $G$ on $\mathbb{R}^{k}$ and free action of $G$ on $\Omega$. First, it is easy to see that Theorem 4.1 is still true. If $\operatorname{dim} G>k$ then $\mathcal{F}_{G}[\Omega]$ is trivial (see [1, Theorem 3.6]) and if $\operatorname{dim} G=k$ and $G$ is biorientable then the formula (4.1) holds (with similar proof). However, if $\operatorname{dim} G=k$ and $G$ is not biorientable then the right-hand side of (4.1) is equal to $\sum_{\alpha} A_{\alpha}$, where $A_{\alpha}$ is either $\mathbb{Z}$ or $\mathbb{Z}_{2}$ depending on whether the respective component $E_{\alpha}$ of $E$ is orientable as a manifold or not. Finally, so far we are not able to give a similar description of $\mathcal{F}_{G}[\Omega]$ if $\operatorname{dim} G<k$ according to Remark 2.4.

\section{The Hopf TYPE THEOREM FOR EQUiVARIANT LOCAL MAPS}

Assume that $V$ is a real finite dimensional orthogonal representation of a compact Lie group $G$ and $\Omega$ is an open invariant subset of $\mathbb{R}^{k} \oplus V$. Let

$$
\Phi_{k}(G)=\{(H) \in \Phi(G) \mid \operatorname{dim} W H \leq k\} .
$$

It is well-known that the set $\operatorname{Iso}(\Omega)$ is finite and so is $\operatorname{Iso}(\Omega) \cap \Phi_{k}(G)$. The following splitting result was proved in [1] and [4].

Theorem 5.1. There are bijections

$$
\begin{aligned}
& \mathcal{F}_{G}[\Omega] \approx \prod_{(H)} \mathcal{F}_{W H}\left[\Omega_{H}\right], \\
& \mathcal{P}_{G}[\Omega] \approx \prod_{(H)} \mathcal{P}_{W H}\left[\Omega_{H}\right],
\end{aligned}
$$

where the products are taken over the set $\operatorname{Iso}(\Omega) \cap \Phi_{k}(G)$. 
We can now formulate the main result of this paper, which may be viewed as an equivariant version of the well-known Hopf theorem. Assume that $V$ is a real finite dimensional orthogonal representation of a compact Lie group $G$ and $\Omega$ is an open invariant subset of $V$.

Theorem 5.2. If $0 \notin \Omega$ or $\operatorname{dim} V^{G}>0$, then there is a natural bijection

$$
\mathcal{F}_{G}[\Omega] \approx \prod_{(H)}\left(\sum_{i=1}^{n(H)} \mathbb{Z}\right),
$$

where the product is taken over the set $\operatorname{Iso}(\Omega) \cap \Phi_{0}(G)$ and the respective direct sums are indexed by the either finite or countably infinite sets of connected components of the quotients $\Omega_{H} / W H$.

Proof. From (5.1) we obtain a product decomposition of $\mathcal{F}_{G}[\Omega]$ with respect to the conjugacy classes from $\operatorname{Iso}(\Omega) \cap \Phi_{0}(G)$ and Theorem 4.3 gives a full description of each factor of this product decomposition, which completes the proof.

We end the paper with some remarks and open problems.

Remark 5.3. It should be emphasized that, apart from the otopy invariance and the Hopf property, the bijection from Theorem 5.2 satisfies also other properties of the topological degree, which is an immediate consequence of our construction. Let us formulate, as an example, the additivity and existence properties. Namely, if we denote the above bijection by deg, then

- $\operatorname{deg}([f \sqcup g])=\operatorname{deg}([f])+\operatorname{deg}([g])$ for $f, g \in \mathcal{F}_{G}(\Omega)$ such that $D_{f} \cap$ $D_{g}=\emptyset$,

- $\operatorname{deg}([f]) \neq 0$ implies $f(x)=0$ for some $x \in \Omega$.

Remark 5.4. Observe that the extreme case of the trivial action covers the classical Hopf theorem. Namely, if $G$ acts trivially on $V$ and $\Omega$ is an open connected subset of $V$, then

$$
\mathcal{F}_{G}[\Omega]=\mathcal{F}_{\{e\}}[\Omega] \approx \mathbb{Z}
$$

Remark 5.5. Of course, Theorem 5.2 remains true if we replace $\mathcal{F}_{G}[\Omega]$ by $\mathcal{P}_{G}[\Omega]$.

Remark 5.6. Although in the parametrized case the splitting formula (5.1) still holds, but for now in this product splitting we can only describe factors for which $\operatorname{dim} W H=k$ as we mentioned at the end of the previous section.

Acknowledgements. The author wishes to express his thanks to the referee for helpful comments concerning the paper. 


\section{REFERENCES}

[1] P. Bartłomiejczyk, On the space of equivariant local maps, Topol. Methods Nonlinear Anal. 45 (2015), 233-246.

[2] P. Bartłomiejczyk, P. Nowak-Przygodzki, Gradient otopies of gradient local maps, Fund. Math. 214 (2011), 89-100.

[3] G. E. Bredon, Topology and Geometry, Springer-Verlag, New York, 1993.

[4] K. Gęba, M. Izydorek, On relations between gradient and classical equivariant homotopy groups of spheres, J. Fixed Point Theory Appl. 12 (2012), 49-58.

[5] K. Gęba, W. Krawcewicz, J. Wu, An equivariant degree with applications to symmetric bifurcation problems I: Construction of the degree, Bull. London. Math. Soc. 69 (1994), 377-398.

[6] V. Guillemin, A. Pollack, Differential topology, Prentice-Hall, 1974.

Faculty of Applied Physics and Mathematics, Gdańsk University of Technology, Gabriela Narutowicza 11/12, 80-233 Gdańsk, Poland

E-mail address: pbartlomiejczyk@mif.pg.gda.pl 